\title{
Coupled coincidence point theorems for compatible mappings in ordered uniform space
}

Duran Turkoglu and Demet Binbasioglu 


\title{
COUPLED COINCIDENCE POINT THEOREMS FOR COMPATIBLE MAPPINGS IN ORDERED UNIFORM SPACE
}

\author{
DURAN TURKOGLU AND DEMET BINBASIOGLU
}

Received 24 September, 2013

\begin{abstract}
In this paper, we use the order relation on uniform spaces defined by [5] to introduce the notion of compatibility of mappings in an ordered uniform space and use this notion to establish coupled coincidence point theorems to ordered uniform space. An example is also given.
\end{abstract}

2010 Mathematics Subject Classification: 54H25; 47H10

Keywords: coupled fixed point, non-linear contractions, ordered uniform space

\section{INTRODUCTION}

There exists considerable literature of fixed point theory dealing with results on fixed or common fixed points in uniform space (e.g. [1-3, 5, 16,21]). But the majority of these results are proved for contractive or contractive type mapping (notice from the cited references). Recently, Aamri and El Moutawakil [1] have introduced the concept of $E$-distance function on uniform spaces and utilize it to improve some well known results of the existing literature involving both $E$-contractive or $E$ - expansive mappings. Lately, I. Altun and M. Imdad [5] have introduced a partial ordering on uniform spaces utilizing $E$ - distance function and have used the same to prove a fixed point theorem for single-valued non-decreasing mappings on ordered uniform spaces. The Banach contraction principle is the most celebrated fixed point theorem. Boyd and Wong [7] extended the Banach contraction principle to the case of nonlinear contraction mappings. Afterward Ciric and Lakshmikantham $[9,13]$ obtained important fixed point theorems. Recently Bhaskar and Lakshmikantham [6], B. S. Choudhury and A. Kundu [8], Nieto and Lopez [14, 15], Ran and Reurings [20] and Agarwal, El-Gebeily and O'Regan [4] presented some new results for contractions in partially ordered metric spaces. Common fixed point results for commuting mappings in metric spaces were first deduced by Jungck [10]. The concept of commuting has been weakened in various directions and in several ways over the years. One such notion which is weaker than commuting is the concept of compatibility introduced by Jungck [11]. Lately, Petre and Páles [18,19] gived fixed point theorems in $E$-Banach 
spaces and $E$-metric spaces. In common fixed point problems, this concept and its generalizations have been used extensively $[12,17]$.

In this paper, we use the partial ordering on uniform spaces which is defined by [5], so we proved some coupled coincidence and coupled common fixed point theorems for a pair of mappings. We also discuss an example.

Now, we mention some relevant definitions and properties from the foundation of uniform spaces. We call a pair $(X, \vartheta)$ to be a uniform space which consists of a non-empty set $X$ together with a uniformity $\vartheta$ wherein the latter begins with a special kind of filter on $X \times X$ whose all elements contain the diagonal $\Delta=\{(x, x): x \in X\}$. If $V \in \vartheta$ and $(x, y) \in V,(y, x) \in V$ then $x$ and $y$ are said to be $V$-close. Also a sequence $\left\{x_{n}\right\}$ in $X$, is said to be a Cauchy sequence with regard to uniformity $\vartheta$ if for any $V \in \vartheta$, there exists $N \geq 1$ such that $x_{n}$ and $x_{m}$ are $V$-close for $m, n \geq N$. A uniformity $\vartheta$ defines a unique topology $\tau(\vartheta)$ on $X$ for which the neighborhoods of $x \in X$ are the sets $V(x)=\{y \in X:(x, y) \in V\}$ when $V$ runs over $\vartheta$.

A uniform space $(X, \vartheta)$ is said to be Hausdorff if and only if the intersection of all the $V \in \vartheta$ reduces to diagonal $\Delta$ of $X$ i.e. $(x, y) \in V$ for $V \in \vartheta$ implies $x=y$. Notice that Hausdorffness of the topology induced by the uniformity guarantees the uniqueness of limit of a sequence in uniform spaces. An element of uniformity $\vartheta$ is said to be symmetrical if $V=V^{-1}=\{(y, x):(x, y) \in V\}$. Since each $V \in \vartheta$ contains a symmetrical $W \in \vartheta$ and if $(x, y) \in W$ then $x$ and $y$ are both $W$ and $V$-close and then one may assume that each $V \in \vartheta$ is symmetrical. When topological concepts are mentioned in the context of a uniform space $(X, \vartheta)$, they are naturally interpreted with respect to the topological space $(X, \tau(\vartheta))$.

\section{PReliminaries}

We shall require the following definitions and lemmas in the sequel.

Definition 1 ([1]). Let $(X, \vartheta)$ be a uniform space. A function $p: X \times X \rightarrow \mathbb{R}^{+}$is said to be an $E$-distance implies

$\left(p_{1}\right)$ For any $V \in \vartheta$ there exists $\delta>0$ such that $p(z, x) \leq \delta$ and $p(z, y) \leq \delta$ for some $z \in X$, imply $(x, y) \in V$,

$\left(p_{2}\right) \quad p(x, y) \leq p(x, z)+p(z, y)$, for any $x, y, z \in X$.

The following lemma embodies some useful properties of $E$-distance.

Lemma $1([1,2])$. Let $(X, \vartheta)$ be a Hausdorff uniform space and $p$ be an Edistance on $X$. Let $\left\{x_{n}\right\}$ and $\left\{y_{n}\right\}$ be arbitrary sequences in $X$ and $\left\{\alpha_{n}\right\},\left\{\beta_{n}\right\}$ be sequences in $\mathbb{R}^{+}$converging to 0 . Then, for $x, y, z \in X$, the following holds:

(a) If $p\left(x_{n}, y\right) \leq \alpha_{n}$ and $p\left(x_{n}, z\right) \leq \beta_{n}$ for all $n \in \mathbb{N}$, then $y=z$. In particular, if $p(x, y)=0$ and $p(x, z)=0$, then $y=z$.

(b) If $p\left(x_{n}, y_{n}\right) \leq \alpha_{n}$ and $p\left(x_{n}, z\right) \leq \beta_{n}$ for all $n \in \mathbb{N}$, then $\left\{y_{n}\right\}$ converges to $z$. 
(c) If $p\left(x_{n}, x_{m}\right) \leq \alpha_{n}$ for all $m>n$, then $\left\{x_{n}\right\}$ is a $p$-Cauchy sequence in $(X, \vartheta)$.

Let $(X, \vartheta)$ be a uniform space equipped with $E$-distance $p$. A sequence in $X$ is $p$-Cauchy if it satisfies the usual metric condition. There are several concepts of completeness in this setting.

Definition $2([1,2])$. Let $(X, \vartheta)$ be a uniform space and $p$ be an $E$-distance on $X$. Then

(i) $X$ said to be $S$-complete if for every $p$-Cauchy sequence $\left\{x_{n}\right\}$ there exists $x \in X$ with $\lim _{n \rightarrow \infty} p\left(x_{n}, x\right)=0$,

(ii) $X$ is said to be $p$-Cauchy complete if for every $p$-Cauchy sequence $\left\{x_{n}\right\}$ there exists $x \in X$ with $\lim _{n \rightarrow \infty} x_{n}=x$ with respect to $\tau(\vartheta)$,

(iii) $f: X \rightarrow X$ is $p$-continuous if $\lim _{n \rightarrow \infty} p\left(x_{n}, x\right)=0$ implies

$\lim _{n \rightarrow \infty} p\left(f x_{n}, f x\right)=0$,

(iv) $f: X \rightarrow X$ is $\tau(\vartheta)$-continuous if $\lim _{n \rightarrow \infty} x_{n}=x$ with respect to $\tau(\vartheta)$ implies $\lim _{n \rightarrow \infty} f x_{n}=f x$ with respect to $\tau(\vartheta)$.

Remark 1 ([1]). Let $(X, \vartheta)$ be a Hausdorff uniform space and let $\left\{x_{n}\right\}$ be a $p$ Cauchy sequence. Suppose that $X$ is $S$-complete, then there exists $x \in X$ such that $\lim _{n \rightarrow \infty} p\left(x_{n}, x\right)=0$. Then Lemma 1 (b) gives that $\lim _{n \rightarrow \infty} x_{n}=x$ with respect to the topology $\tau(\vartheta)$ which shows that $S$-completeness implies $p$-Cauchy completeness.

Lemma 2 ([5]). Let $(X, \vartheta)$ be a Hausdorff uniform space, $p$ be E-distance on $X$ and $\varphi: X \rightarrow \mathbb{R}$. Define the relation" $\preceq$ " on $X$ as follows;

$$
x \preceq y \Leftrightarrow x=y \text { or } p(x, y) \leq \varphi(x)-\varphi(y) .
$$

Then " $\preceq "$ is a (partial) order on $X$ induced by $\varphi$.

Definition 3 ([6]). We call an element $(x, y) \in X \times X$ a coupled fixed point of the mapping $T$ if $T(x, y)=x, T(y, x)=y$.

Definition 4 ([13]). An element $(x, y) \in X \times X$ is called a coupled coincidence point of a mapping $T: X \times X \rightarrow X$ and $g: X \rightarrow X$ implies

$T(x, y)=g(x), T(y, x)=g(y)$.

Definition 5 ([13]). Let $X$ be a non-empty set and $T: X \times X \rightarrow X$ and $g: X \rightarrow X$. We say $T$ and $g$ are commutative implies

$g(T(x, y))=T(g(x), g(y))$

for any $x, y \in X$.

Definition 6 ([8]). The mappings $T$ and $g$ where $T: X \times X \rightarrow X$ and $g: X \rightarrow X$, are said to be compatible implies

$$
\lim _{n \rightarrow \infty} p\left(g\left(T\left(x_{n}, y_{n}\right)\right), T\left(g\left(x_{n}\right), g\left(y_{n}\right)\right)\right)=0
$$


and

$\lim _{n \rightarrow \infty} p\left(g\left(T\left(y_{n}, x_{n}\right)\right), T\left(g\left(y_{n}\right), g\left(x_{n}\right)\right)\right)=0$

whenever $\left\{x_{n}\right\}$ and $\left\{y_{n}\right\}$ are sequences in $X$, such that

$\lim _{n \rightarrow \infty} T\left(x_{n}, y_{n}\right)=\lim _{n \rightarrow \infty} g\left(x_{n}\right)=x$ and $\lim _{n \rightarrow \infty} T\left(y_{n}, x_{n}\right)=\lim _{n \rightarrow \infty} g\left(y_{n}\right)=y$, for any $x, y \in X$ are satisfied.

\section{MAIN RESULTS}

Definition 7. Let $(X, \vartheta)$ be a uniform space and let " $\preceq$ " be an order relation on $X$ and let $T: X \times X \rightarrow X$ be an operator. We say that $T$ has the mixed monotone property if $T(x, y)$ is monotone nondecreasing in $x$ and is monotone nonincreasing in $y$, that is for any $x, y \in X$,

$$
x_{1}, x_{2} \in X, x_{1} \preceq x_{2} \Rightarrow T\left(x_{1}, y\right) \preceq T\left(x_{2}, y\right)
$$

and

$$
y_{1}, y_{2} \in X, y_{1} \preceq y_{2} \Rightarrow T\left(x, y_{1}\right) \succeq T\left(x, y_{2}\right) .
$$

Definition 8. Let $(X, \vartheta)$ be a uniform space and let " $\preceq$ " be an order relation on $X$ and let $T: X \times X \rightarrow X, g: X \rightarrow X$ be operators. We say $T$ has the mixed $g$-monotone property if $T$ is monotone $g$-non-decreasing in its first argument and is monotone $g-$ non-increasing in its second argument, that is, for any $x, y \in X$,

$x_{1}, x_{2} \in X, g\left(x_{1}\right) \preceq g\left(x_{2}\right)$ implies

$$
T\left(x_{1}, y\right) \preceq T\left(x_{2}, y\right)
$$

and

$y_{1}, y_{2} \in X, g\left(y_{1}\right) \preceq g\left(y_{2}\right)$ implies

$$
T\left(x, y_{1}\right) \succeq T\left(x, y_{2}\right)
$$

If $g$ is the identity mapping, then Definition 8 reduces to Definition 7 .

Theorem 1. Let $(X, \vartheta)$ be a uniform space, " $\preceq "$ is an order on $X$ and suppose there is an $E$-distance $p$ on $X$ such that $(X, p)$ is a $p$-Cauchy complete uniform space. Let $T: X \times X \rightarrow X$ be a $\tau(\vartheta)$ continuous mapping having the mixed monotone property on $X$. Assume that there exists a $k \in[0,1)$ with

$p(T(x, y), T(u, v)) \leq \frac{k}{2}[p(x, u)+p(y, v)]$

for all comparable $x, u$ and all comparable $y$, $v$. If there exist $x_{0}, y_{0} \in X$ such that

$x_{0} \preceq T\left(x_{0}, y_{0}\right)$ and $y_{0} \succeq T\left(y_{0}, x_{0}\right)$,

then there exist $x, y \in X$ such that $x=T(x, y)$ and $y=T(x, y)$.

Proof. Since $x_{0} \preceq T\left(x_{0}, y_{0}\right)=x_{1}$ and $y_{0} \succeq T\left(x_{0}, y_{0}\right)=y_{1}$

letting $x_{2}=T\left(x_{1}, y_{1}\right)$ and $y_{2}=T\left(y_{1}, x_{1}\right)$, we denote 
$T^{2}\left(x_{0}, y_{0}\right)=T\left(T\left(x_{0}, y_{0}\right), T\left(y_{0}, x_{0}\right)\right)=T\left(x_{1}, y_{1}\right)=x_{2}$.

$T^{2}\left(y_{0}, x_{0}\right)=T\left(T\left(y_{0}, x_{0}\right), T\left(x_{0}, y_{0}\right)\right)=T\left(y_{1}, x_{1}\right)=y_{2}$.

With this notation, we now have, due to the mixed monotone property of $T$,

$x_{2}=T^{2}\left(x_{0}, y_{0}\right)=T\left(x_{1}, y_{1}\right) \succeq T\left(x_{0}, y_{0}\right)=x_{1}$ and

$y_{2}=T^{2}\left(y_{0}, x_{0}\right)=T\left(y_{1}, x_{1}\right) \preceq T\left(y_{0}, x_{0}\right)=y_{1}$.

Further, for $n=1,2, \ldots$ we let

$x_{n+1}=T^{n+1}\left(x_{0}, y_{0}\right)=T\left(T^{n}\left(x_{0}, y_{0}\right), T^{n}\left(y_{0}, x_{0}\right)\right)$

and

$y_{n+1}=T^{n+1}\left(y_{0}, x_{0}\right)=T\left(T^{n}\left(y_{0}, x_{0}\right), T^{n}\left(x_{0}, y_{0}\right)\right)$.

We can easily verify that

$x_{0} \preceq T\left(x_{0}, y_{0}\right)=x_{1} \preceq T^{2}\left(x_{0}, y_{0}\right)=x_{2} \preceq \ldots \preceq T^{n+1}\left(x_{0}, y_{0}\right) \preceq \ldots$

and

$y_{0} \succeq T\left(y_{0}, x_{0}\right)=y_{1} \succeq T^{2}\left(y_{0}, x_{0}\right)=y_{2} \succeq \ldots \succeq T^{n+1}\left(y_{0}, x_{0}\right) \succeq \ldots$

Now, we claim that, for $n \in \mathbb{N}$,

$p\left(T^{n+1}\left(x_{0}, y_{0}\right), T^{n}\left(x_{0}, y_{0}\right)\right) \leq \frac{k^{n}}{2}\left[p\left(T\left(x_{0}, y_{0}\right), x_{0}\right)+p\left(T\left(y_{0}, x_{0}\right), y_{0}\right)\right]$

$p\left(T^{n+1}\left(y_{0}, x_{0}\right), T^{n}\left(y_{0}, x_{0}\right)\right) \leq \frac{k^{n}}{2}\left[p\left(T\left(y_{0}, x_{0}\right), y_{0}\right)+p\left(T\left(x_{0}, y_{0}\right), x_{0}\right)\right]$

Indeed, for $n=1$, using $T\left(x_{0}, y_{0}\right) \succeq x_{0}$ and $T\left(y_{0}, x_{0}\right) \preceq y_{0}$, we get

$p\left(T^{2}\left(x_{0}, y_{0}\right), T\left(x_{0}, y_{0}\right)\right)=p\left(T\left(T\left(x_{0}, y_{0}\right), T\left(y_{0}, x_{0}\right)\right), T\left(x_{0}, y_{0}\right)\right)$

Similarly,

$$
\leq \frac{k}{2}\left[p\left(T\left(x_{0}, y_{0}\right), x_{0}\right)+p\left(T\left(y_{0}, x_{0}\right), y_{0}\right)\right] .
$$

$$
\begin{gathered}
p\left(T^{2}\left(y_{0}, x_{0}\right), T\left(y_{0}, x_{0}\right)\right)=p\left(T\left(T\left(y_{0}, x_{0}\right), T\left(x_{0}, y_{0}\right)\right), T\left(y_{0}, x_{0}\right)\right) \\
=p\left(T\left(y_{0}, x_{0}\right), T\left(T\left(y_{0}, x_{0}\right), T\left(x_{0}, y_{0}\right)\right)\right) \\
\leq \frac{k}{2}\left[p\left(T\left(x_{0}, y_{0}\right), x_{0}\right)+p\left(T\left(y_{0}, x_{0}\right), y_{0}\right)\right] .
\end{gathered}
$$

Now, assume that $(i)$ and $(i i)$ hold. Using

$$
\begin{gathered}
T^{n+1}\left(x_{0}, y_{0}\right) \succeq T^{n}\left(x_{0}, y_{0}\right) \text { and } T^{n+1}\left(y_{0}, x_{0}\right) \preceq T^{n}\left(y_{0}, x_{0}\right) \text { we get } \\
p\left(T^{n+2}\left(x_{0}, y_{0}\right), T^{n+1}\left(x_{0}, y_{0}\right)\right)=p\left(T\left(T^{n+1}\left(x_{0}, y_{0}\right), T^{n+1}\left(y_{0}, x_{0}\right)\right)\right. \\
\left.\quad, T\left(T^{n}\left(x_{0}, y_{0}\right), T^{n}\left(y_{0}, x_{0}\right)\right)\right) \\
\leq \frac{k}{2}\left[p\left(T^{n+1}\left(x_{0}, y_{0}\right), T^{n}\left(x_{0}, y_{0}\right)\right)+p\left(T^{n+1}\left(y_{0}, x_{0}\right), T^{n}\left(y_{0}, x_{0}\right)\right)\right] \\
\leq \frac{k^{n+1}}{2}\left[p\left(T\left(x_{0}, y_{0}\right), x_{0}\right)+p\left(T\left(y_{0}, x_{0}\right), y_{0}\right)\right] .
\end{gathered}
$$

Similarly, one can show that

$p\left(T^{n+2}\left(y_{0}, x_{0}\right), T^{n+1}\left(y_{0}, x_{0}\right)\right) \leq \frac{k^{n+1}}{2}\left[p\left(T\left(y_{0}, x_{0}\right), y_{0}\right)+p\left(T\left(x_{0}, y_{0}\right), x_{0}\right)\right]$. This implies that $\left\{T^{n}\left(x_{0}, y_{0}\right)\right\}$ and $\left\{T^{n}\left(y_{0}, x_{0}\right)\right\}$ are $p$-Cauchy sequences in $X$.

Indeed, let $m>n$, then

$$
\begin{aligned}
p\left(T^{m}\left(x_{0}, y_{0}\right), T^{n}\left(x_{0}, y_{0}\right)\right) & \leq p\left(T^{m}\left(x_{0}, y_{0}\right), T^{m-1}\left(x_{0}, y_{0}\right)\right)+ \\
& \ldots+p\left(T^{n+1}\left(x_{0}, y_{0}\right), T^{n}\left(x_{0}, y_{0}\right)\right) \\
\leq & \frac{k^{m-1}+\ldots+k^{n}}{2}\left[p\left(T\left(x_{0}, y_{0}\right), x_{0}\right)+p\left(T\left(y_{0}, x_{0}\right), y_{0}\right)\right] \\
\leq & \frac{k^{n}}{2(1-k)}\left[p\left(T\left(x_{0}, y_{0}\right), x_{0}\right)+p\left(T\left(y_{0}, x_{0}\right), y_{0}\right)\right] .
\end{aligned}
$$

Similarly, we can verify that $\left\{T^{n}\left(y_{0}, x_{0}\right)\right\}$ is also a $p$-Cauhy sequence.

Since $X$ is a $p-$ Cauchy complete uniform space, there exist $x, y \in X$ such that $\lim _{n \rightarrow \infty} T^{n}\left(x_{0}, y_{0}\right)=x$ and $\lim _{m \rightarrow \infty} T^{m}\left(y_{0}, x_{0}\right)=y$. 
Finally, we claim $T(x, y)=x$ and $T(y, x)=y$.

Let $\varepsilon>0$. Since $T$ is $\tau(\vartheta)$-continuous at $(x, y)$, for a given $\frac{\varepsilon}{2}>0$, there exists a $\delta>0$ such that $p(x, u)+p(y, v)<\delta$ implies $p(T(x, y), T(u, v))<\frac{\varepsilon}{2}$.

Since $\left\{T^{n}\left(x_{0}, y_{0}\right)\right\} \rightarrow x$ and $\left\{T^{n}\left(y_{0}, x_{0}\right)\right\} \rightarrow y$, for $\eta=\min \left(\frac{\varepsilon}{2}, \frac{\delta}{2}\right)>0$, there exist $n_{0}, m_{0}$ such that, for $n \geq n_{0}, m \geq m_{0}$,

$p\left(T^{n}\left(x_{0}, y_{0}\right), x\right)<\eta$ and $p\left(T^{m}\left(y_{0}, x_{0}\right), y\right)<\eta$.

Now, for $n \in \mathbb{N}, n \geq \max \left\{n_{0}, m_{0}\right\}$,

$$
\begin{aligned}
p(T(x, y), x) & \leq p\left(T(x, y), T^{n+1}\left(x_{0}, y_{0}\right)\right)+p\left(T^{n+1}\left(x_{0}, y_{0}\right), x\right) \\
& =p\left(T(x, y), T\left(T^{n}\left(x_{0}, y_{0}\right), T^{n}\left(y_{0}, x_{0}\right)\right)\right)+p\left(T^{n+1}\left(x_{0}, y_{0}\right), x\right) \\
& <\frac{\varepsilon}{2}+\eta \leq \varepsilon .
\end{aligned}
$$

This implies that $T(x, y)=x$. Similarly, we can show that $T(y, x)=y$.

Theorem 2. Let $(X, \vartheta)$ be a uniform space, " $\preceq "$ is an order on $X$ and suppose there is an $E$-distance $p$ on $X$ such that $(X, p)$ is a $p$-Cauchy complete uniform space. Assume there is a function $\phi:[0, \infty) \rightarrow[0, \infty)$ with $\phi(t)<t$ and $\lim _{r \rightarrow t+} \phi(r)<$ $t$ for each $t>0$ and also suppose $T: X \times X \rightarrow X$ and $g: X \rightarrow X$ are such that $T$ has the mixed $g-$ monotone property and

$$
p(T(x, y), T(u, v)) \leq \phi\left(\frac{p(g(x), g(u))+p(g(y), g(v))}{2}\right)
$$

for all $x, y, u, v \in X$ for which $g(x), g(u)$ are comparable and $g(y), g(v)$ are comparable. Suppose

$T(X \times X) \subseteq g(X), g$ is $\tau(\vartheta)$ - continuous and monotone increasing and $T$ and $g$ be compatible mappings. Also suppose

(a) $T$ is $\tau(\vartheta)$ - continuous or

(b) $X$ has the following property:

(i) if a non-decreasing sequence $\left\{x_{n}\right\} \rightarrow x$, then

$$
x_{n} \preceq x
$$

for all $n$,

(ii) if a non-increasing sequence $\left\{y_{n}\right\} \rightarrow y$, then

$$
y \preceq y_{n}
$$

for all $n$.

If there exist $x_{0}, y_{0} \in X$ such that

$g\left(x_{0}\right) \preceq T\left(x_{0}, y_{0}\right)$ and $g\left(y_{0}\right) \succeq T\left(y_{0}, x_{0}\right)$,

then there exist $x, y \in X$ such that

$g(x)=T(x, y)$ and $g(y)=T(y, x)$,

that is, $T$ and $g$ have a coupled coincidence point in $X$. 
Proof. Let $x_{0}, y_{0} \in X$, be such that $g\left(x_{0}\right) \preceq T\left(x_{0}, y_{0}\right)$ and $g\left(y_{0}\right) \succeq T\left(y_{0}, x_{0}\right)$. Since

$T(X \times X) \subseteq g(X)$, we can define $x_{1}, y_{1} \in X$ such that $g\left(x_{1}\right)=T\left(x_{0}, y_{0}\right)$ and $g\left(y_{1}\right)=T\left(y_{0}, x_{0}\right)$.

In the same way we construct,

$g\left(x_{2}\right)=T\left(x_{1}, y_{1}\right)$ and $g\left(y_{2}\right)=T\left(y_{1}, x_{1}\right)$.

Continuing in this way we construct two sequences $\left\{g\left(x_{n}\right)\right\}$ and $\left\{g\left(y_{n}\right)\right\}$ in $X$ such that,

$g\left(x_{n+1}\right)=T\left(x_{n}, y_{n}\right)$ and

$$
g\left(y_{n+1}\right)=T\left(y_{n}, x_{n}\right)
$$

for all $n \geq 0$.

Now we prove that for all $n \geq 0$,

$$
g\left(x_{n}\right) \preceq g\left(x_{n+1}\right)
$$

and

$$
g\left(y_{n}\right) \succeq g\left(y_{n+1}\right) .
$$

Since $g\left(x_{0}\right) \preceq T\left(x_{0}, y_{0}\right)$ and $g\left(y_{0}\right) \succeq T\left(y_{0}, x_{0}\right)$, in view of $g\left(x_{1}\right)=T\left(x_{0}, y_{0}\right)$ and $g\left(y_{1}\right)=T\left(y_{0}, x_{0}\right)$, we have $g\left(x_{0}\right) \preceq g\left(x_{1}\right)$ and $g\left(y_{0}\right) \succeq g\left(y_{1}\right)$, that is, (3.9) and (3.10) hold for $n=0$.

We presume that (3.9) and (3.10) hold for some $n>0$. As $T$ has the mixed $g$-monotone property and $g\left(x_{n}\right) \preceq g\left(x_{n+1}\right), g\left(y_{n}\right) \succeq g\left(y_{n+1}\right)$, from (8), we get $g\left(x_{n+1}\right)=T\left(x_{n}, y_{n}\right) \preceq T\left(x_{n+1}, y_{n}\right)$ and

$$
T\left(y_{n+1}, x_{n}\right) \preceq T\left(y_{n}, x_{n}\right)=g\left(y_{n+1}\right) .
$$

Also for the same reason we have $g\left(x_{n+2}\right)=T\left(x_{n+1}, y_{n+1}\right) \succeq T\left(x_{n+1}, y_{n}\right)$ and

$$
T\left(y_{n+1}, x_{n}\right) \succeq T\left(y_{n+1}, x_{n+1}\right)=g\left(y_{n+2}\right) .
$$

Then from (3.11) and (3.12)

$$
g\left(x_{n+1}\right) \preceq g\left(x_{n+2}\right) \text { and } g\left(y_{n+1}\right) \succeq g\left(y_{n+2}\right) \text {. }
$$

Then, by mathematical induction it follows that (3.9) and (3.10) hold for all $n \geq 0$. Let, $\delta_{n}=p\left(g\left(x_{n}\right), g\left(x_{n+1}\right)\right)+p\left(g\left(y_{n}\right), g\left(y_{n+1}\right)\right)$ and $\delta_{n}^{1}=p\left(g\left(x_{n+1}\right), g\left(x_{n}\right)\right)+$ $p\left(g\left(y_{n+1}\right), g\left(y_{n}\right)\right)$.

Next we prove that

$\delta_{n} \leq 2 \phi\left(\frac{\delta_{n-1}}{2}\right)$ and

$$
\delta_{n}^{\prime} \leq 2 \phi\left(\frac{\delta_{n-1}^{1}}{2}\right) .
$$

Since for all $n \geq 0, g\left(x_{n-1}\right) \preceq g\left(x_{n}\right)$ and $g\left(y_{n-1}\right) \succeq g\left(y_{n}\right)$, we have from (3.5) and (3.8),

$$
p\left(g\left(x_{n}\right), g\left(x_{n+1}\right)\right)=p\left(T\left(x_{n-1}, y_{n-1}\right), T\left(x_{n}, y_{n}\right)\right)
$$




$$
\leq \phi\left(\frac{p\left(g\left(x_{n-1}\right), g\left(x_{n}\right)\right)+p\left(g\left(y_{n-1}\right), g\left(y_{n}\right)\right)}{2}\right)=\phi\left(\frac{\delta_{n-1}}{2}\right)
$$

and

$$
\begin{aligned}
& p\left(g\left(x_{n+1}\right), g\left(x_{n}\right)\right)=p\left(T\left(x_{n}, y_{n}\right), T\left(x_{n-1}, y_{n-1}\right)\right) \\
& \quad \leq \phi\left(\frac{p\left(g\left(x_{n}\right), g\left(x_{n-1}\right)\right)+p\left(g\left(y_{n}\right), g\left(y_{n-1}\right)\right)}{2}\right)=\phi\left(\frac{\delta_{n-1}^{\prime}}{2}\right) .
\end{aligned}
$$

Similarly from (3.5) and (3.8), we have for all $n \geq 0$,

$$
\begin{aligned}
& p\left(g\left(y_{n}\right), g\left(y_{n+1}\right)\right)=p\left(T\left(y_{n-1}, x_{n-1}\right), T\left(y_{n}, x_{n}\right)\right) \\
& \quad \leq \phi\left(\frac{p\left(g\left(y_{n-1}\right), g\left(y_{n}\right)\right)+p\left(g\left(x_{n-1}\right), g\left(x_{n}\right)\right)}{2}\right)=\phi\left(\frac{\delta_{n-1}}{2}\right)
\end{aligned}
$$

and

$$
\begin{aligned}
& p\left(g\left(y_{n+1}\right), g\left(y_{n}\right)\right)=p\left(T\left(y_{n}, x_{n}\right), T\left(y_{n-1}, x_{n-1}\right)\right) \\
& \quad \leq \phi\left(\frac{p\left(g\left(y_{n}\right), g\left(y_{n-1}\right)\right)+p\left(g\left(x_{n}\right), g\left(x_{n-1}\right)\right)}{2}\right)=\phi\left(\frac{\delta_{n-1}^{\prime}}{2}\right) .
\end{aligned}
$$

Combining (3.14) and (3.15) we obtain(3.13).

Since $\phi(t)<t$ for $t>0$, it follows from (3.13) that the sequences $\left\{\delta_{n}\right\}$ and $\left\{\delta_{n}^{1}\right\}$ are monotone decreasing sequence of non-negative real numbers. Hence there exist $\delta \geq 0$ and $\delta^{l} \geq 0$ such that $\lim _{n \rightarrow \infty} \delta_{n}=\delta$ and $\lim _{n \rightarrow \infty} \delta_{n}^{l}=\delta^{l}$. If possible, let $\delta>0$. Taking the limit as $n \rightarrow \infty$ in (3.13) and using $\lim _{r \rightarrow t^{+}} \phi(r)<t$ for all $t>0$, we obtain

$$
\delta=\lim _{n \rightarrow \infty} \delta_{n} \leq 2 \lim _{n \rightarrow \infty} \phi\left(\frac{\delta_{n-1}}{2}\right)=2 \lim _{\delta_{n-1} \rightarrow \delta^{+}} \phi\left(\frac{\delta_{n-1}}{2}\right)<2 \frac{\delta}{2}=\delta,
$$

which is a contradiction. Thus $\delta=0$. Hence we have

$$
\lim _{n \rightarrow \infty}\left[p\left(g\left(x_{n}\right), g\left(x_{n+1}\right)\right)+p\left(g\left(y_{n}\right), g\left(y_{n+1}\right)\right)\right]=\lim _{n \rightarrow \infty} \delta_{n}=0
$$

and similarly $\delta^{\prime}=0$ that is $\lim _{n \rightarrow \infty}\left[p\left(g\left(x_{n+1}\right), g\left(x_{n}\right)\right)+p\left(g\left(y_{n+1}\right), g\left(y_{n}\right)\right)\right]=$ $\lim _{n \rightarrow \infty} \delta_{n}^{1}=0$.

Next we show that $\left\{g\left(x_{n}\right)\right\}$ and $\left\{g\left(y_{n}\right)\right\}$ are p-Cauchy sequences. Let at least one of $\left\{g\left(x_{n}\right)\right\}$ and $\left\{g\left(y_{n}\right)\right\}$ be not a $p-C a u c h y$ sequence. Then there exists $\varepsilon>0$ and sequences of natural numbers $\{m(k)\}$ and $\{l(k)\}$ such that for every natural number $k$

$$
\begin{aligned}
& m(k)>l(k) \geq k \text { and } \\
& \quad p_{k}=p\left(g\left(x_{l(k)}\right), g\left(x_{m(k)}\right)\right)+p\left(g\left(y_{l(k)}\right), g\left(y_{m(k)}\right)\right) \geq \varepsilon .
\end{aligned}
$$

Now corresponding to $l(k)$ we can choose $m(k)$ to be the smallest positive integer for which (3.17) holds. Then,

$$
p\left(g\left(x_{l(k)}\right), g\left(x_{m(k)-1}\right)\right)+p\left(g\left(y_{l(k)}\right), g\left(y_{m(k)-1}\right)\right)<\varepsilon .
$$

Further from (3.17) and (3.18), for all $k \geq 0$, we have,

$$
\varepsilon \leq p_{k} \leq p\left(g\left(x_{l(k)}\right), g\left(x_{m(k)-1}\right)\right)+p\left(g\left(x_{m(k)-1}\right), g\left(x_{m(k)}\right)\right)
$$


$+p\left(g\left(y_{l(k)}\right), g\left(y_{m(k)-1}\right)\right)+p\left(g\left(y_{m(k)-1}\right), g\left(y_{m(k)}\right)\right)$

$=p\left(g\left(x_{l(k)}\right), g\left(x_{m(k)-1}\right)\right)+p\left(g\left(y_{l(k)}\right), g\left(y_{m(k)-1}\right)\right)+\delta_{m(k)-1}<\varepsilon+\delta_{m(k)-1}$.

Taking the limit as $k \rightarrow \infty$, we have by (3.16),

$$
\lim _{k \rightarrow \infty} p_{k}=\varepsilon
$$

Again, for all $k \geq 0$, we have,

$$
\begin{aligned}
& p_{k}=p\left(g\left(x_{l(k)}\right), g\left(x_{m(k)}\right)\right)+p\left(g\left(y_{l(k)}\right), g\left(y_{m(k)}\right)\right) \\
& \quad \leq p\left(g\left(x_{l(k)}\right), g\left(x_{l(k)+1}\right)\right) \\
& \quad+p\left(g\left(x_{l(k)+1}\right), g\left(x_{m(k)+1}\right)\right)+p\left(g\left(x_{m(k)+1}\right), g\left(x_{m(k)}\right)\right) \\
& \quad+p\left(g\left(y_{l(k)}\right), g\left(y_{l(k)+1}\right)\right) \\
& \quad+p\left(g\left(y_{l(k)+1}\right), g\left(y_{m(k)+1}\right)\right)+p\left(g\left(y_{m(k)+1}\right), g\left(y_{m(k)}\right)\right) \\
& \quad=p\left(g\left(x_{l(k)}\right), g\left(x_{l(k)+1}\right)\right)+p\left(g\left(y_{l(k)}\right), g\left(y_{l(k)+1}\right)\right) \\
& \quad+p\left(g\left(x_{l(k)+1}\right), g\left(x_{m(k)+1}\right)\right) \\
& \quad+p\left(g\left(y_{l(k)+1}\right), g\left(y_{m(k)+1}\right)+p\left(g\left(x_{m(k)+1}\right), g\left(x_{m(k)}\right)\right)\right. \\
& +p\left(g\left(y_{m(k)+1}\right), g\left(y_{m(k)}\right)\right) .
\end{aligned}
$$

Hence, for all $k \geq 0$

$$
p_{k} \leq \delta_{l(k)}+\delta_{m(k)}^{1}+p\left(g\left(x_{l(k)+1}\right), g\left(x_{m(k)+1}\right)\right)+p\left(g\left(y_{l(k)+1}\right), g\left(y_{m(k)+1}\right)\right) \text {. }
$$

From (3.5),(3.8),(3.9),(3.10) and (3.17), for all $k \geq 0$, we obtain

$$
\begin{aligned}
& p\left(g\left(x_{l(k)+1}\right), g\left(x_{m(k)+1}\right)\right)=p\left(T\left(x_{l(k)}, y_{l(k)}\right), T\left(x_{m(k)}, y_{m(k)}\right)\right) \\
& \leq \phi\left(\frac{p\left(g\left(x_{l(k)}\right), g\left(x_{m(k)}\right)\right)+p\left(g\left(y_{l(k)}\right), g\left(y_{m(k)}\right)\right)}{2}\right)=\phi\left(\frac{p_{k}}{2}\right) .
\end{aligned}
$$

Also by (3.5),(3.8),(3.9),(3.10) and (3.17), for all $k \geq 0$, we have,

$$
\begin{aligned}
& p\left(g\left(y_{l(k)+1}\right), g\left(y_{m(k)+1}\right)\right)=p\left(T\left(y_{l(k)}, x_{l(k)}\right), T\left(y_{m(k)}, x_{m(k)}\right)\right) \\
& \leq \phi\left(\frac{p\left(g\left(x_{l(k)}\right), g\left(x_{m(k)}\right)\right)+p\left(g\left(y_{l(k)}\right), g\left(y_{m(k)}\right)\right)}{2}\right)=\phi\left(\frac{p_{k}}{2}\right) .
\end{aligned}
$$

Putting (3.21) and (3.22) in (3.20) for all $k \geq 0$, we obtain, $p_{k} \leq \delta_{l(k)}+\delta_{m(k)}^{1}+$ $2 \phi\left(\frac{p_{k}}{2}\right)$.

Letting $n \rightarrow \infty$ in the above inequality and using (3.16),(3.17) and (3.19) we obtain,

$$
\varepsilon \leq 2 \lim _{k \rightarrow \infty} \phi\left(\frac{p_{k}}{2}\right)=2 \lim _{p_{k} \rightarrow \varepsilon^{+}} \phi\left(\frac{p_{k}}{2}\right)<2 \frac{\varepsilon}{2}=\varepsilon,
$$

which is a contradiction. Therefore, $\left\{g\left(x_{n}\right)\right\}$ and $\left\{g\left(y_{n}\right)\right\}$ are p-Cauchy sequences in $X$ and hence they are convergent in the p-Cauchy complete uniform space $(X, \vartheta)$. Let 


$$
\begin{aligned}
& \lim _{n \rightarrow \infty} T\left(x_{n}, y_{n}\right)=\lim _{n \rightarrow \infty} g\left(x_{n}\right)=x \text { and } \\
& \lim _{n \rightarrow \infty} T\left(y_{n}, x_{n}\right)=\lim _{n \rightarrow \infty} g\left(y_{n}\right)=y .
\end{aligned}
$$

Since $T$ and $g$ are compatible mappings, we have by (3.24),

$$
\lim _{n \rightarrow \infty} p\left(g\left(T\left(x_{n}, y_{n}\right)\right), T\left(g\left(x_{n}\right), g\left(y_{n}\right)\right)\right)=0
$$

and

$$
\lim _{n \rightarrow \infty} p\left(g\left(T\left(y_{n}, x_{n}\right)\right), T\left(g\left(y_{n}\right), g\left(x_{n}\right)\right)\right)=0 .
$$

Next we prove that $g(x)=T(x, y)$ and $g(y)=T(y, x)$.

Let $(a)$ hold.

For all $n \geq 0$, we have,

$$
\begin{gathered}
p\left(g\left(x_{n}\right), T\left(g\left(x_{n}\right), g\left(y_{n}\right)\right)\right) \\
\leq p\left(g\left(x_{n}\right), g\left(T\left(x_{n}, y_{n}\right)\right)\right)+p\left(g\left(T\left(x_{n}, y_{n}\right)\right), T\left(g\left(x_{n}\right), g\left(y_{n}\right)\right)\right) .
\end{gathered}
$$

Taking the limit as $n \rightarrow \infty$, using (3.8), (3.24),(3.25) and the fact that $T$ and $g$ are continuous, we have $p\left(g\left(x_{n}\right), T(x, y)\right)=0$.

Similarly, from (3.8), (3.24),(3.26) and the continuities of $T$ and $g$, we have $p\left(g\left(y_{n}\right), T(y, x)\right)=0$.

Combining the above two results we get $g(x)=T(x, y)$ and $g(y)=T(y, x)$.

Next we suppose that $(b)$ holds.

By (3.9), (3.10) and (3.24) we have $\left.\left\{g\left(x_{n}\right)\right\}\right\}$ is non-decreasing sequence, $g\left(x_{n}\right) \rightarrow$ $x$ and $\left\{g\left(y_{n}\right)\right\}$ is non-increasing sequence,

$g\left(y_{n}\right) \rightarrow y$ as $n \rightarrow \infty$. Then by (3.6) and (3.7) we have for all $n \geq 0$,

$$
g\left(x_{n}\right) \preceq x \text { and } g\left(y_{n}\right) \succeq y .
$$

Since, $T$ and $g$ are compatible mappings and $g$ is continuous, by (3.25) and (3.26) we have,

$$
\lim _{n \rightarrow \infty} g\left(g\left(x_{n}\right)\right)=g(x)=\lim _{n \rightarrow \infty} g\left(T\left(x_{n}, y_{n}\right)\right)=\lim _{n \rightarrow \infty} T\left(g\left(x_{n}\right), g\left(y_{n}\right)\right)
$$

and

$$
\lim _{n \rightarrow \infty} g\left(g\left(y_{n}\right)\right)=g(y)=\lim _{n \rightarrow \infty} g\left(T\left(y_{n}, x_{n}\right)\right)=\lim _{n \rightarrow \infty} T\left(g\left(y_{n}\right), g\left(x_{n}\right)\right) .
$$

Now we have $p(g(x), T(x, y)) \leq p\left(g(x), g\left(g x_{n+1}\right)\right)+p\left(g\left(g\left(x_{n+1}\right)\right), T(x, y)\right)$.

Taking the limit as $n \rightarrow \infty$ in the above inequality, using (3.8) and (3.28) we have,

$$
\begin{aligned}
& p(g(x), T(x, y)) \\
\leq & \lim _{n \rightarrow \infty} p\left(g(x), g\left(g\left(x_{n+1}\right)\right)\right)+\lim _{n \rightarrow \infty} p\left(g\left(T\left(x_{n}, y_{n}\right)\right), T(x, y)\right) \\
\leq & \lim _{n \rightarrow \infty} p\left(T\left(g\left(x_{n}\right), g\left(y_{n}\right)\right), T(x, y)\right) .
\end{aligned}
$$

Since the mapping $g$ is monotone increasing, by (3.5), (3.27) and the above inequality, we have for all $n \geq 0$, 
$p(g(x), T(x, y)) \leq \lim _{n \rightarrow \infty} \phi\left(\frac{p\left(g\left(g\left(x_{n}\right)\right), g(x)\right)+p\left(g\left(g\left(y_{n}\right)\right), g(y)\right)}{2}\right)$.

Using (3.24) and the property of $\phi$ - function we obtain, $p(g(x), T(x, y)) \leq 0$.

That is

$g(x)=T(x, y)$

and similarly, by virtue of (3.8), (3.24) and (3.29) we obtain

$g(y)=T(y, x)$.

Thus we have proved that $T$ and $g$ have coupled coincidence point in $X$.

This completes the proof of the Theorem 2.

Remark 2. If we assume $g(x)=x$ for all $x \in X$ and $\phi(t)=k t$ for $0 \leq k<1$ then we obtain the results of Theorem 1 .

Corollary 1. Let $(X, \vartheta)$ be a uniform space, " $\preceq "$ is an order on $X$ and suppose there is an $E$-distance $p$ on $X$ such that $(X, p)$ is a $p$-Cauchy complete uniform space. Assume there is a function $\phi:[0, \infty) \rightarrow[0, \infty)$ with $\phi(t)<t$ and $\lim _{r \rightarrow t+} \phi(r)<$ $t$ for each $t>0$ and also suppose $T: X \times X \rightarrow X$ and $g: X \rightarrow X$ are such that $T$ has the mixed $g$-monotone property and

$$
p(T(x, y), T(u, v)) \leq \phi\left(\frac{p(g(x), g(u))+p(g(y), g(v))}{2}\right)
$$

for all $x, y, u, v \in X$ for which comparable $g(x), g(u)$ and comparable $g(y), g(v)$. Suppose

$T(X \times X) \subseteq g(X), g$ is $\tau(\vartheta)-$ continuous and commutes with $T$ and also suppose either

(a) $T$ is $\tau(\vartheta)$ - continuous or

(b) $X$ has the following property:

(i) if a non-decreasing sequence $\left\{x_{n}\right\} \rightarrow x$, then

$$
x_{n} \preceq x
$$

for all $n$,

(ii) if a non-increasing sequence $\left\{y_{n}\right\} \rightarrow y$, then

$$
y \preceq y_{n}
$$

for all $n$.

If there exist $x_{0}, y_{0} \in X$ such that

$g\left(x_{0}\right) \preceq T\left(x_{0}, y_{0}\right)$ and $g\left(y_{0}\right) \succeq T\left(y_{0}, x_{0}\right)$,

then there exist $x, y \in X$ such that

$g(x)=T(x, y)$ and $g(y)=T(y, x)$,

that is, $T$ and $g$ have a coupled coincidence.

Example 1. Let $X=[0,1] \times[0,1], p(x, y)=\left|x_{1}-x_{2}\right|+\left|y_{1}-y_{2}\right|$ for $x=$ $\left(x_{1}, x_{2}\right), y=\left(y_{1}, y_{2}\right) \in X$ and " $\preceq$ " is a partially ordered with the natural ordering of 
real numbers. Then $(X, \preceq)$ be an ordered uniform space and $(X, p)$ is a $p-$ Cauchy complete uniform space. Let $g: X \rightarrow X$ be defined as $g(x)=x^{2}=\left(x_{1}^{2}, x_{2}^{2}\right)$ for all $x=\left(x_{1}, x_{2}\right) \in X$.

Let $T: X \times X \rightarrow X$ be defined as;

$T(x, y)=\left\{\begin{array}{cc}\left(\frac{\left(x_{1}-y_{1}\right)^{2}}{3}, \frac{\left(x_{2}-y_{2}\right)^{2}}{3}\right) & x, y \in X, x \succeq y \\ (0,0), & x \prec y\end{array}\right.$

$T$ corresponds the mixed $g$-monotone property.

Let $\phi:[0, \infty) \rightarrow[0, \infty)$ be defined as

$\phi(t)=\frac{2}{3} t$, for $t \in[0, \infty)$.

Let $\left\{x_{n}\right\}$ and $\left\{y_{n}\right\}$ be two sequences in $X$ such that,

$\lim _{n \rightarrow \infty} T\left(x_{n}, y_{n}\right)=a, \lim _{n \rightarrow \infty} g\left(x_{n}\right)=a, a=\left(a_{1}, a_{2}\right)$

$\lim _{n \rightarrow \infty} T\left(y_{n}, x_{n}\right)=b, \lim _{n \rightarrow \infty} g\left(y_{n}\right)=b, b=\left(b_{1}, b_{2}\right)$.

Then obviously, $a=(0,0)$ and $b=(0,0)$.

Now, for all $n \geq 0$;

$$
\begin{aligned}
& g\left(x_{n}\right)=x_{n}^{2}=\left(x_{n(1)}^{2}, x_{n(2)}^{2}\right), x_{n}=\left(x_{n(1)}, x_{n(2)}\right) \in X \\
& g\left(y_{n}\right)=y_{n}^{2}=\left(y_{n(1)}^{2}, y_{n(2)}^{2}\right), y_{n}=\left(y_{n(1)}, y_{n(2)}\right) \in X \\
& T\left(x_{n}, y_{n}\right)=\left\{\begin{array}{cc}
\left(\frac{x_{n(1)}^{2}-y_{n(1)}^{2}}{3}, \frac{x_{n(2)}^{2}-y_{n(2)}^{2}}{3}\right), & \text { if } x_{n} \succeq y_{n} \\
(0,0), & x_{n} \prec y_{n}
\end{array}\right. \\
& T\left(y_{n}, x_{n}\right)=\left\{\begin{array}{cc}
\left(\frac{y_{n(1)}^{2}-x_{n(1)}^{2}}{3}, \frac{y_{n(2)}^{2}-x_{n(2)}^{2}}{3}\right), & \text { if } y_{n} \succeq x_{n} \\
(0,0), & y_{n} \prec x_{n}
\end{array}\right.
\end{aligned}
$$

Then, it follows that;

$\lim _{n \rightarrow \infty} p\left(g\left(T\left(x_{n}, y_{n}\right)\right), T\left(g\left(x_{n}\right), g\left(y_{n}\right)\right)\right) \rightarrow 0$ as $n \rightarrow \infty$ and

$\lim _{n \rightarrow \infty} p\left(g\left(T\left(y_{n}, x_{n}\right)\right), T\left(g\left(y_{n}\right), g\left(x_{n}\right)\right)\right) \rightarrow 0$ as $n \rightarrow \infty$.

Hence, the mappings $T$ and $g$ are compatible in $X$.

Also, $x_{0}=0=(0,0)$ and $y_{0}=c=\left(c_{1}, c_{2}\right)$ are two points in $X$ such that $g\left(x_{0}\right)=g((0,0))=(0,0)=T\left((0,0),\left(c_{1}, c_{2}\right)\right)=T\left(x_{0}, y_{0}\right)$

and

$g\left(y_{0}\right)=g\left(\left(c_{1}, c_{2}\right)\right)=\left(c_{1}^{2}, c_{2}^{2}\right) \succeq\left(\frac{c_{1}^{2}}{3}, \frac{c_{2}^{2}}{3}\right)=T\left(\left(c_{1}, c_{2}\right),(0,0)\right)=T\left(y_{0}, x_{0}\right)$.

We next verify inequality (5) of Theorem 2 . We take $x, y, u, v \in X$, such that $g(x)=g\left(\left(x_{1}, x_{2}\right)\right) \preceq g\left(\left(u_{1}, u_{2}\right)\right)=g(u)$ and $g(y) \succeq g(v)$, that is, $x^{2} \preceq u^{2} \Rightarrow$ $x_{1}^{2} \preceq u_{1}^{2}$ and $x_{2}^{2} \preceq u_{2}^{2}$ and $y^{2} \succeq v^{2} \Rightarrow y_{1}^{2} \succeq v_{1}^{2}$ and $y_{2}^{2} \succeq v_{2}^{2}$.

We consider the following cases:

Case 1: $x \succeq y$ and $u \succeq v$.

Then $p(T(x, y), T(u, v))=p\left(\left(\frac{x_{1}^{2}-y_{1}^{2}}{3}, \frac{x_{2}^{2}-y_{2}^{2}}{3}\right),\left(\frac{u_{1}^{2}-v_{1}^{2}}{3}, \frac{u_{2}^{2}-v_{2}^{2}}{3}\right)\right)$ 
Case 2: $x \succeq y$ and $u \prec v$.

$$
\begin{aligned}
& =\left|\frac{x_{1}^{2}-y_{1}^{2}}{3}-\frac{u_{1}^{2}-v_{1}^{2}}{3}\right|+\left|\frac{x_{2}^{2}-y_{2}^{2}}{3}-\frac{u_{2}^{2}-v_{2}^{2}}{3}\right| \\
& =\left|\frac{x_{1}^{2}-u_{1}^{2}}{3}-\frac{y_{1}^{2}-v_{1}^{2}}{3}\right|+\left|\frac{x_{2}^{2}-u_{2}^{2}}{3}-\frac{y_{2}^{2}-v_{2}^{2}}{3}\right| \\
& \preceq\left(\left|\frac{x_{1}^{2}-u_{1}^{2}}{3}\right|+\left|\frac{x_{2}^{2}-u_{2}^{2}}{3}\right|\right)+\left(\left|\frac{y_{1}^{2}-v_{1}^{2}}{3}\right|+\left|\frac{y_{2}^{2}-v_{2}^{2}}{3}\right|\right) \\
& =\frac{2}{3}\left\{\frac{\left|x_{1}^{2}-u_{1}^{2}\right|+\left|x_{2}^{2}-u_{2}^{2}\right|}{2}+\frac{\left|y_{1}^{2}-v_{1}^{2}\right|+\left|y_{2}^{2}-v_{2}^{2}\right|}{2}\right\} \\
& =\phi\left(\frac{p((g(x), g(u)))+p((g(y), g(v)))}{2}\right) .
\end{aligned}
$$

Then $p(T(x, y), T(u, v))=p\left(\left(\frac{x_{1}^{2}-y_{1}^{2}}{3}, \frac{x_{2}^{2}-y_{2}^{2}}{3}\right),(0,0)\right)$

$$
\begin{aligned}
& =\left|\frac{x_{1}^{2}-y_{1}^{2}}{3}\right|+\left|\frac{x_{2}^{2}-y_{2}^{2}}{3}\right| \\
& =\left|\frac{u_{1}^{2}+x_{1}^{2}-y_{1}^{2}-u_{1}^{2}}{3}\right|+\left|\frac{u_{2}^{2}+x_{2}^{2}-y_{2}^{2}-u_{2}^{2}}{3}\right| \\
& =\left|\frac{\left(u_{1}^{2}-y_{1}^{2}\right)-\left(u_{1}^{2}-x_{1}^{2}\right)}{3}\right|+\left|\frac{\left(u_{2}^{2}-y_{2}^{2}\right)-\left(u_{2}^{2}-x_{2}^{2}\right)}{3}\right| \\
& \left.\preceq\left|\frac{\left(v_{1}^{2}-y_{1}^{2}\right)+\left(u_{1}^{2}-x_{1}^{2}\right)}{3}\right|+\left|\frac{\left(v_{2}^{2}-y_{2}^{2}\right)+\left(u_{2}^{2}-x_{2}^{2}\right)}{3}\right| \text { (since } v \succ u\right) \\
& \preceq\left(\left|\frac{u_{1}^{2}-x_{1}^{2}}{3}\right|+\left|\frac{u_{2}^{2}-x_{2}^{2}}{3}\right|\right)+\left(\left|\frac{v_{1}^{2}-y_{1}^{2}}{3}\right|+\left|\frac{v_{2}^{2}-y_{2}^{2}}{3}\right|\right) \\
& =\frac{2}{3}\left\{\frac{\left|x_{1}^{2}-u_{1}^{2}\right|+\left|x_{2}^{2}-u_{2}^{2}\right|}{2}+\frac{\left|y_{1}^{2}-v_{1}^{2}\right|+\left|y_{2}^{2}-v_{2}^{2}\right|}{2}\right\} \\
& =\phi\left(\frac{p(g(x), g(u)))+p((g(y), g(v)))}{2}\right) .
\end{aligned}
$$

Case 3: $x \prec y$ and $u \succeq v$.

Then $p(T(x, y), T(u, v))=p\left((0,0),\left(\frac{u_{1}^{2}-v_{1}^{2}}{3}, \frac{u_{2}^{2}-v_{2}^{2}}{3}\right)\right)$

$$
\begin{aligned}
& =\left|\frac{u_{1}^{2}-v_{1}^{2}}{3}\right|+\left|\frac{u_{2}^{2}-v_{2}^{2}}{3}\right| \\
& =\left|\frac{u_{1}^{2}+x_{1}^{2}-v_{1}^{2}-x_{1}^{2}}{3}\right|+\left|\frac{u_{2}^{2}+x_{2}^{2}-v_{2}^{2}-x_{2}^{2}}{3}\right| \\
& =\left|\frac{\left(x_{1}^{2}-v_{1}^{2}\right)-\left(u_{1}^{2}-x_{1}^{2}\right)}{3}\right|+\left|\frac{\left(x_{2}^{2}-v_{2}^{2}\right)-\left(u_{2}^{2}-x_{2}^{2}\right)}{3}\right| \\
& \left.\preceq\left|\frac{\left(y_{1}^{2}-v_{1}^{2}\right)+\left(u_{1}^{2}-x_{1}^{2}\right)}{3}\right|+\left|\frac{\left(y_{2}^{2}-v_{2}^{2}\right)+\left(u_{2}^{2}-x_{2}^{2}\right)}{3}\right| \text { (since } y \succ x\right) \\
& \preceq\left(\left|\frac{u_{1}^{2}-x_{1}^{2}}{3}\right|+\left|\frac{u_{2}^{2}-x_{2}^{2}}{3}\right|\right)+\left(\left|\frac{y_{1}^{2}-v_{1}^{2}}{3}\right|+\left|\frac{y_{2}^{2}-v_{2}^{2}}{3}\right|\right)
\end{aligned}
$$




$$
\begin{aligned}
& =\frac{2}{3}\left\{\frac{\left|x_{1}^{2}-u_{1}^{2}\right|+\left|x_{2}^{2}-u_{2}^{2}\right|}{2}+\frac{\left|y_{1}^{2}-v_{1}^{2}\right|+\left|y_{2}^{2}-v_{2}^{2}\right|}{2}\right\} \\
& =\phi\left(\frac{p((g(x), g(u)))+p((g(y), g(v)))}{2}\right) .
\end{aligned}
$$

Case 4: $x \prec y$ and $u \prec v$ with $x^{2} \preceq u^{2}$ and $y^{2} \succeq v^{2}$.

Then $T(x, y)=0$ and $T(u, v)=0$, that is $p(T(x, y), T(u, v))=0$. Obviously (5) is satisfied.

Thus it is verified that the functions $T, g, \phi$ satisfy all the conditions of Theorem 2. Here $(0,0)$ is the coupled coincidence point of $T$ and $g$ in $X$.

Remark 3. In this example $T$ and $g$ are not commuting maps. Thus the result of [13] which is noted here in Corollary 1 is not applicable to this example.

\section{ACKNOWLEDGEMENT}

We would like to thank the referees for their suggestions that improved the presentation of the paper.

\section{REFERENCES}

[1] M. Aamri and D. El Moutawakil, "Common fixed point theorems for E-contractive or E-expansive maps in uniform spaces," Acta Mathematica Academiae Peadegogicae Nyiregyhaziensis, vol. 20, pp. 83-91, 2004.

[2] M. Aamri and D. El Moutawakil, "Weak compatibility and common fixed point theorems for Acontractive and E-expansive maps in uniform spaces," Serdica Math. J., vol. 31, pp. 75-86, 2005.

[3] R. P. Agarwal, D. O'Regan, and N. S. Papageorgiou, "Common fixed point theory for multi-valued contractive maps of Reich type in uniform spaces," Appl. Anal., vol. 83, no. 1, pp. 37-47, 2004.

[4] R. Agarwal, M. El-Gebeily, and D. O'Regan, "Generalized contractions in partially ordered metric spaces," Appl. Anal., vol. 87, pp. 109-116, 2008.

[5] I. Altun and M. Imdad, "Some fixed point theorems on ordered uniform spaces," Filomat, vol. 23, no. 3, pp. 15-22, 2009.

[6] T. Bhaskar and V. Lakshmikantham, "Fixed point theorems in partially ordered metric spaces and applications," Nonlinear Anal., vol. 65, pp. 1379-1393, 2006.

[7] D. Boyd and J. Wong, "On nonlinear contractions," Proc. Amer. Math. Soc., vol. 20, pp. 458-464, 1969.

[8] B. S. Choudhury and A. Kundu, "A coupled coincidence point result in partially ordered metric spaces for compatible mappings," Nonlinear Analysis, vol. 73, pp. 2524-2531, 2010.

[9] L. Ciric and V. Lakshmikantham, "Coupled random fixed point theorems for nonlinear contractions in partially ordered metric spaces," Stoch. Anal. Appl., vol. 27, no. 6, pp. 1246-1259, 2009.

[10] G. Jungck, "Commuting mappings and fixed points," Amer. Math. Monthly, vol. 83, pp. 261-263, 1976.

[11] G. Jungck, "Compatible mappings and common fixed points," Int. J. Math. Math. Sci., vol. 9, pp. 771-779, 1986.

[12] S. Kang, Y. Cho, and G. Jungck, "Common fixed point of compatible mappings," Int. J. Math. Math. Sci., vol. 13, no. 1, pp. 61-66, 1990.

[13] V. Lakshmikantham and L. Ciric, "Coupled fixed point theorems for nonlinear contractions in partially ordered metric spaces," Nonlinear Anal., vol. 70, pp. 4341-4349, 2009. 
[14] J. J. Nieto and R. R. Lopez, "Contractive mapping theorems in partially ordered sets and applications to ordinary differential equations," Order, vol. 22, pp. 223-239, 2005.

[15] J. J. Nieto and R. R. Lopez, "Existence and uniqueness of fixed point in partially ordered sets and applications to ordinary differential equations," Acta Math. Sinica Engl. Ser., vol. 23, no. 12, pp. 2205-2212, 2007.

[16] M. O. Olatinwo, "On some common fixed point theorems of Aamri and El Moutawakil in uniform spaces," Appilied Mathematics E-Notes, vol. 8, pp. 254-262, 2008.

[17] S. Padaliya and R. Pant, "Common fixed point for R-weakly commuting mapping of type (Af )," Soochow J. Math., vol. 2, pp. 145-163, 2005.

[18] Z. Páles and I.-R. Petre, "Iterative fixed point theorems in E-metric spaces," Acta Math. Hung., vol. 140, no. 1-2, pp. 134-144, 2013.

[19] I.-R. Petre, "Fixed points for $\varphi$-contractions in E-banach spaces," Fixed Point Theory, vol. 13, no. 2, pp. 623-640, 2012.

[20] A. Ran and M. Reurings, "A fixed point theorem in partially ordered sets and some applications to matrix equations," Proc. Amer. Math. Soc., vol. 132, pp. 1435-1443, 2004.

[21] D. Turkoglu and D. Binbasioglu, "Some fixed point theorems for multivalued monotone mappings in ordered uniform space," Fixed Point Theory and Applications, 2011.

Authors' addresses

Duran Turkoglu

Department of Mathematics, Faculty of Science, University of Gazi, 06500-Teknikokullar, Ankara, TURKEY, (or Department of Mathematics, Faculty of Science and Arts, University of Amasya, Amasya, Turkey)

E-mail address: dturkoglu@gazi.edu.tr

\section{Demet Binbasioglu}

Department of Mathematics, Faculty of Science, University of Gazi, 06500-Teknikokullar, Ankara, Turkey

E-mail address: demetbinbasi@gazi.edu.tr 